\title{
Self-adaptive e-Learning Website for Mathematics
}

\author{
Akira Nakamura
}

\begin{abstract}
Keyword searching and browsing on learning website is ultimate self-adaptive learning. Our e-learning website "KIT Mathematics Navigation" for mathematics is already optimized for keyword search. We added two functions on our website: displaying inbound links on top of the webpages and the button on top of the webpages to access the graph drawing of knowledge structure of mathematics. The combination of two functions enables learners to make their own learning path not only towards basic mathematical knowledge but also towards advanced mathematical knowledge just by clicking hyperlinks while checking the importance and hierarchy of mathematics knowledge via graph drawing of knowledge structure of mathematics.
\end{abstract}

Index Terms-Adaptive learning, hyperlink, knowledge structure, mathematics.

\section{INTRODUCTION}

Learning method by using information and communication technology (ICT), so called e-learning, is widely used in not only at education institutions and businesses but also in individual lives. There are many sorts of e-learnings and many ways to categorize them. In the case of focusing on learning strategies, e-learnings were categorized into two types: instructive learning and constructive learning [1], [2]. Massive Open Online Courses (MOOC) [3], [4] like Coursera, Udacity and edXare of particular interest nowadays. MOOC belongs to instructive learning. The traditional step by step learning by using physical text books also belongs to instructive learning. Adaptive e-learning belongs to constructive learning. It also has become the focus of a lot of attention in recent years since it provides the suitable learning materials for the needs and ability of individual learners due to the progress of technology. Many researchers have attempted to make adaptive learning systems which create adaptive learning materials for each learner by analyzing learners' ability under some algorithm [2], [5]. Website whose webpages are connected to each other with hyperlinks provide an adaptive e-learning environment because learners make their own learning path by clicking hyperlinks. Search engines like Google, Bing and Yahoo can be regarded to be the most useful adaptive learning tool. Many people use search engines in studying on a daily basis. Despite that, there are few educational researches related to search engines. Ref. [6]-[8] Learners can access the learning materials they want to learn by using keyword search and by making their own adaptive learning path in the world wide space by themselves.

These two types of learning are complementary to each

Manuscript received May 20, 2015; revised July 20, 2015. This work was supported by JSPS KAKENHI Grant Number 25350359.

A. Nakamura is with the Kanazawa Institute of Technology, Nonoichi, Ishikawa 921-8501, Japan (e-mail: n.akira@ neptune.kanazawa-it.ac.jp). other. For example, when learners read a physical text book and encounter what they are not able to understand, they might use keyword search to understand the content. We focus on the latter type of learning and have been developing the mathematical e-learning website which is suitable for adaptive learning.

\section{DEVELoPING E-LEARNING WEBSITE FOR MATHEMATICS}

\section{A. Framework of e-Learning Website}

We have been developing the e-Learning website "KIT Mathematics Navigation" which is written in Japanese since 2004 [9]. We translate the contents of our website from Japanese to English by using Google Translate [10]. The quality of translation is not sufficient at this stage but might be improved in the future. The concept of our e-learning website is "a mathematics online reference" which is open to the public on the Internet. Our e-learning website consists of two parts. One is a reference part which consists of mathematics knowledge, and the other is an exercise part which consists of many questions and their answers. In the case of web based learning, hyperlink and keyword search are very useful functions to learn efficiently. In order to get the most out of those functions, we structure the website as follows. One page is allocated to a specific mathematical knowledge. The title of the webpage contains a keyword representing the mathematics knowledge. Keywords in a webpage document are linked to related webpages as a reference. The number of webpages in our website is over two thousand. We set a mathematics keyword or a mathematics key phrase which are suitable for the webpage content as the page title in consideration of search engine optimization. Therefore, learners can easily access a webpage on our website by using a keyword search.

\section{B. Link Back Learning}

About $80 \%$ of visitors on our website come via search engines to obtain the information they need [11]. They read the documents in the target webpages they accessed after keyword searching, and then they click the hyperlinks on the webpages when they want to read reference webpages. Visitors make their own learning paths by clicking the hyperlinks one after another to understand target mathematical knowledge. Basically the directions of hyperlinks are from webpages whose contents are advanced to webpages whose contents are basic since hyperlinks are created to access reference webpages. We call this type of learning "Link Back Learning" [11]. Fig. 1 illustrates "Link Back Learning" in contrast with traditional "Step by Step Learning." Step by step learning belongs to instructive learning. On the other hand, link back learning belongs to adaptive learning. 
Under the framework of our website it is difficult for learners to make their own learning paths toward advanced materials since hyperlinks in the webpage documents connect to basic webpages, and they have to access advanced webpages via index webpages. We tried to improve our website framework so the learners can access advanced webpages easily. This attempt will be described in the following sections.

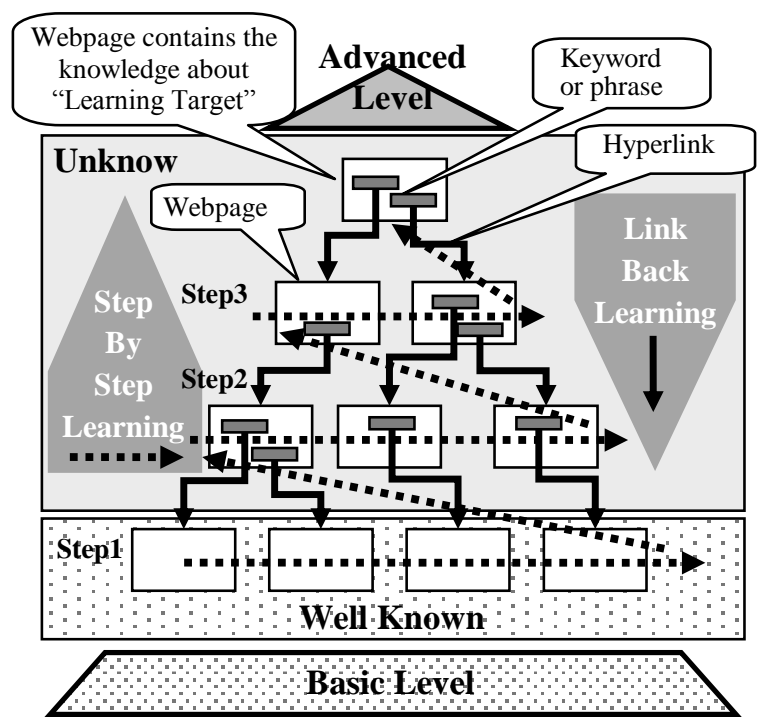

Fig. 1. Illustration of link back learning.

\section{BASIC WORK}

This work is based on my previous works. These are hierarchy construction of mathematical knowledge [12] and a graph drawing of mathematics knowledge [13], [14].

\section{A. Hierarchy Construction of Mathematical Knowledge}

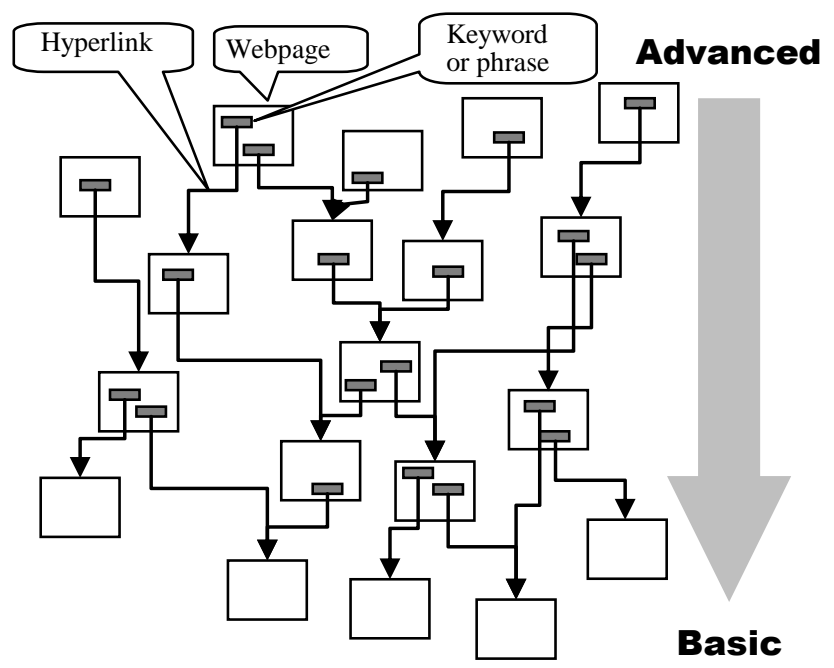

Fig. 2. Diagram of hyperlink structure.

Mathematics knowledge is constructed by stacking advanced knowledge derived from basic knowledge. As a result mathematics knowledge forms a hierarchy structure. In our website, hyperlinks connect two webpages which have a unit of mathematical knowledge. The directions of hyperlinks are from the webpages whose contents are more advanced to the ones whose contents are more basic. Fig. 2 shows the diagram of the hyperlink structure. The hyperlink structure represents the structure of mathematics knowledge. We calculated hierarchy of webpages i.e. mathematical knowledge by using an adjacent matrix under the hypothesis that higher hierarchal mathematical knowledge are related to more closely to higher hierarchical mathematical knowledge than the lower one are related to. 759 pages are listed in the higher hierarchical order. Calculated higher hierarchical order is mostly acceptable. However, some portions of the calculated higher hierarchical order of mathematics knowledge are not acceptable. For example, some of webpages whose contents are related to differential equation are lower than webpages whose contents are related to integral. We analyzed the cause of those issues and found that densities of hyperlinks are not sufficient to calculate the hierarchy. We are now consulting calculated higher hierarchical order and are attempting to improve the hyperlink structure by adding hyperlinks on the webpages whose hierarchical orders are problematic.

\section{B. Graph Drawing of Mathematics Knowledge}

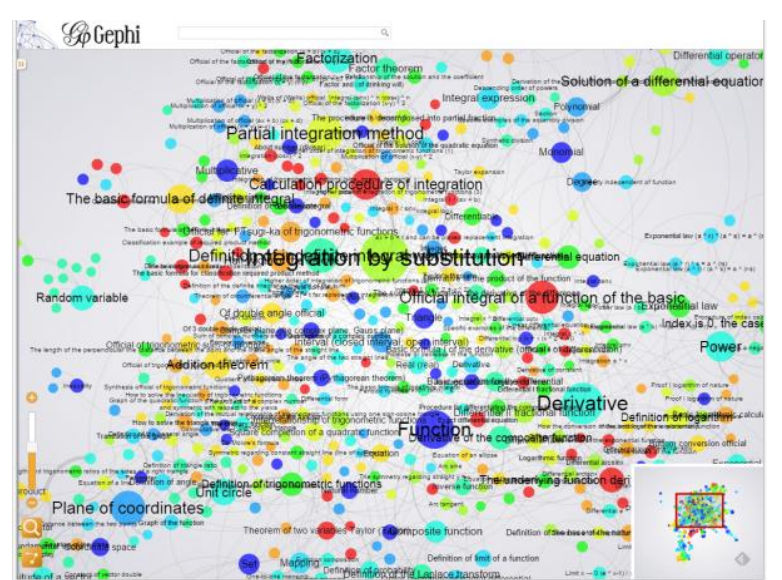

Fig. 3. Graph drawing of knowledge structure of mathematics combined with knowledge level.

We consider that the hyperlink structure resembles the knowledge structure of mathematics. We visualize the knowledge structure by using a graph drawing. In the graph drawing each node indicates a webpage i.e. mathematical knowledge and each edge indicates hyperlink. The hyperlink has directions. Therefore, the graph is a directed graph.

We use the Gephi which is open source software for visualization [15]. We developed crawler software with PHP and MySQL to make the network data for Gephi. We display a graph drawing on a webpage by using the JavaScript GEXF Viewer for Gephi under the MIT license [16]. Fig. 3 shows a capture image of the English version of graph drawing which is already open to public on the Internet [17]. The number of nodes is 759 and the number of edges is 2194 at this stage. The titles of webpages are allocated to node labels. The sizes of nodes (circle) depend on the number of inbound links. Large nodes have many inbound links which means the knowledge has significant importance in mathematical knowledge. There are several layout algorithms to set the graph shape. The graph drawing of Fig. 3 was created by Force Atras in the layout menu of Gephi. The distances between nodes depend on the relationships between two mathematical knowledge. Short distances indicate that two mathematical knowledge are close and similar knowledge 
level. We divided the hierarchy of mathematics knowledge evenly into 11 parts, and allocated a color to each part. 11 colors are 11 gradations from blue to red via green. That is, blue-color nodes indicate the most basic level knowledge group, green-color nodes indicate mid-level knowledge group and red-color nodes indicate the most advanced level knowledge group.

We can intuitively understand the importance of mathematical knowledge by the sizes of nodes and the knowledge level of mathematics by the colors of nodes and also intensities of relationship between two mathematical knowledge by the distances between two nodes.

If we select a node (i.e. a mathematical knowledge on the graph drawing), a side menu appears on the left of the graph drawing. The Java Script GEXF Viewer has a function which displays only the selected node and the ones linked to and from that specific node as shown in Fig. 4. On the side menu, there are hyperlinks connected to the webpage of the selected node, inbound links list and the outbound links list. The inbound links list shows the nodes linking to the selected node which are listed in order of hierarchy. These nodes (i.e. mathematical knowledge) are more advanced knowledge than the selected knowledge. The outbound links list shows nodes linked form selected node which are listed in order of hierarchy. These nodes (i.e. mathematical knowledge) are more basic knowledge than the selected knowledge. We can easily check the content of selected mathematical knowledge to click the hyperlink at the top of the side menu. We add this function into the viewer software. The JavaScript GEXF Viewer has a function to zoom in and zoom out the graph drawing. We can grasp the outline of mathematical knowledge by zooming out the graph drawing, and we can understand the micro structure of mathematics knowledge by zooming in the graph drawing.

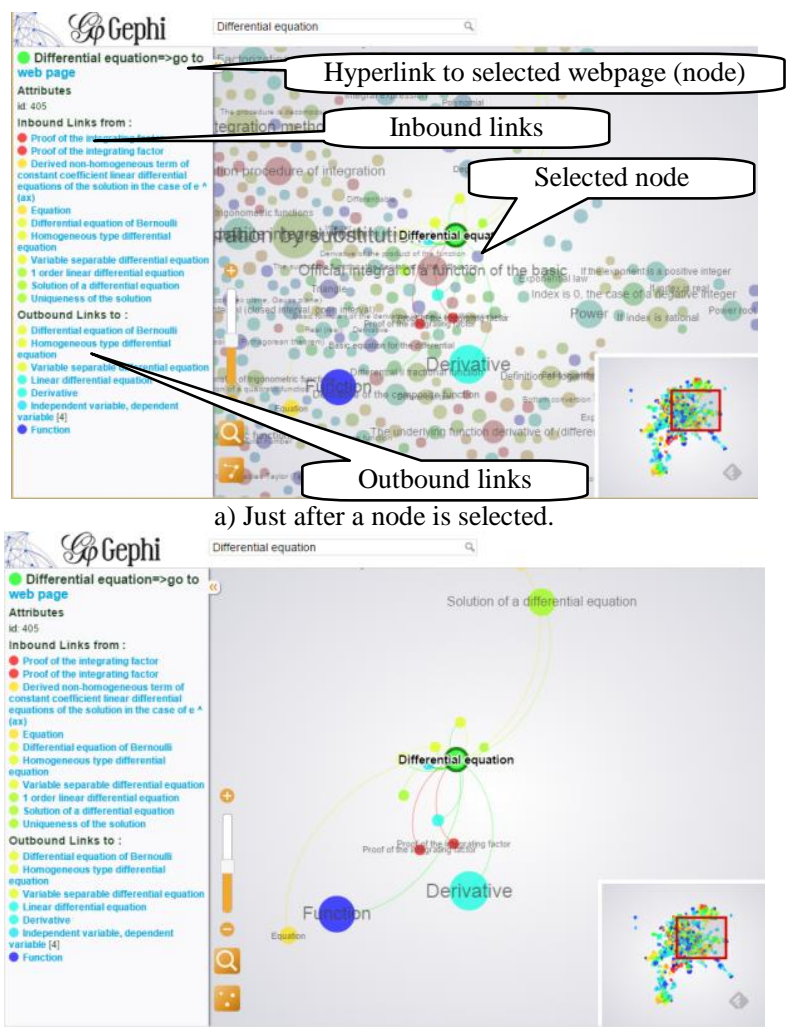

b) A view of selected node and nodes connecting to selected node. Fig. 4. A view of the case when a node is selected.

\section{METHOD OF SELF-ADAPTIVE LEARNING}

An action of keyword searching is based on the learner's will. Decisions whether or not to click hyperlinks depend on the learner's will. Therefore, keyword searching and browsing on learning website is ultimate self-adaptive learning. To optimize self-adaptive learning environment we added two functions on the webpages in our e-learning website for mathematics.

We developed the program to display inbound links on top of webpages based on network data as shown in Fig. 5. These inbound links are hyperlinks connecting to advanced knowledge. These inbound links are listed in order of hierarchy. With the availability of inbound links learners make their own learning path toward not only basic mathematical knowledge but also toward advanced mathematical knowledge simply by clicking hyperlinks as shown in Fig. 6.

We set the button on top of the webpage to access the graph drawing of a knowledge structure of mathematics. If learners click on the button, the graph drawing of a knowledge structure of mathematics with the selected mathematics knowledge which learners are studying at the moment appears. The learners can figure out how important the knowledge is by the size of the node, and how difficult the knowledge is by the color of the node as shown in Fig. 4. This structure allows learners to go on to the next step by selecting links on the left-hand side menu.

The two functions mentioned above enable learners to learn mathematics efficiently and effectively according their ability and curiosity.

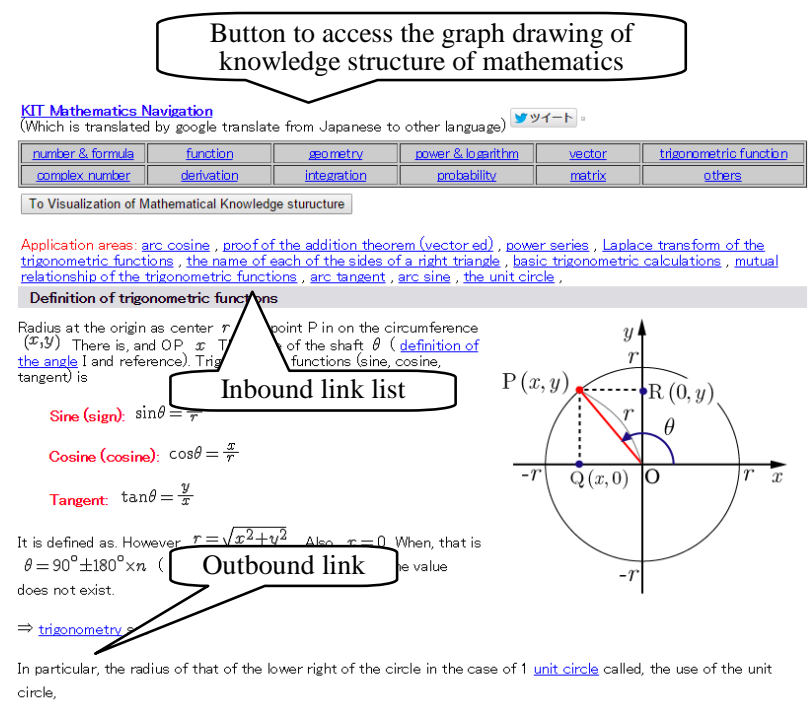

Fig. 5. Screenshot of a webpage.

Fig. 7 shows the screen shot of a question and its answer page in the exercise part. We made hyperlinks on keywords and key phrases of mathematics knowledge in the answer document. Learners can browse reference webpages by clicking hyperlinks if they want to. And then they start self-adaptive learning.

About 8 thousand learners visited our e-learning website for mathematics on weekdays and about 5 thousand learners visited on weekends in May 2015. About $80 \%$ of the visitors accessed via search engines. More than $60 \%$ of visitors are mobile users. 


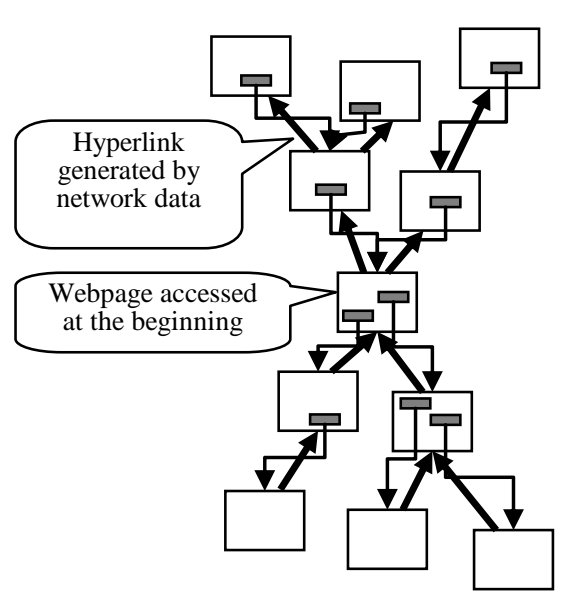

Advanced

Fig. 6. Diagram of hyperlink structure added inbound links.

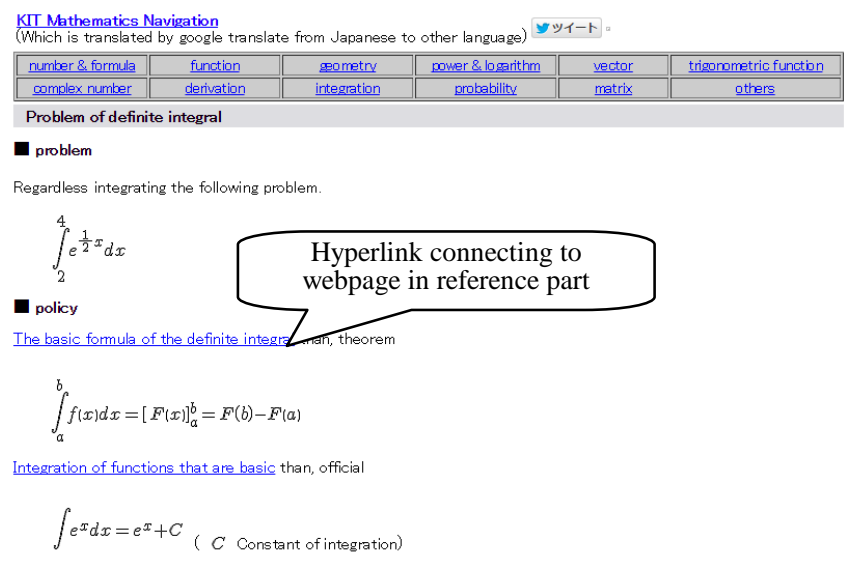

Fig. 7. Screen shot of question and answer webpage.

TABLE I: THE NUMBER OF WEBPAGES LEARNERS ACCESSED IN MAY 2015

\begin{tabular}{|c|c|c|}
\hline page depth & number of session & personage \\
\hline 1 & 150,517 & $72.07 \%$ \\
\hline 2 & 23,693 & $11.34 \%$ \\
\hline 3 & 12,806 & $6.13 \%$ \\
\hline 4 & 5,163 & $2.47 \%$ \\
\hline 5 & 4,245 & $2.03 \%$ \\
\hline 6 & 2,212 & $1.06 \%$ \\
\hline 7 & 2,084 & $1.00 \%$ \\
\hline 8 & 1,154 & $0.55 \%$ \\
\hline 9 & 1,185 & $0.57 \%$ \\
\hline 10 & 753 & $0.36 \%$ \\
\hline 11 & 724 & $0.35 \%$ \\
\hline 12 & 492 & $0.24 \%$ \\
\hline 13 & 534 & $0.26 \%$ \\
\hline 14 & 343 & $0.16 \%$ \\
\hline 15 & 328 & $0.16 \%$ \\
\hline 16 & 229 & $0.11 \%$ \\
\hline 17 & 240 & $0.11 \%$ \\
\hline 18 & 205 & $0.10 \%$ \\
\hline 19 & 187 & $0.09 \%$ \\
\hline $20+$ & 1,756 & $0.84 \%$ \\
\hline
\end{tabular}

Table I shows the number of webpages learners accessed a session in May, 2015. The data is obtained from Google Analytics. About $70 \%$ of the learners just accessed one webpage. But the others made their own learning paths to understand mathematical knowledge.

We can obtain the access log data on how learners make their own learning paths on our website. We consider that it is possible to evaluate the ability and interest, motivation, and attitude toward learning by analyzing access log data in detail. A learner who tends to browse toward advanced mathematics knowledge has a great potential to having good motivation or a keen interest. A learner who tends to browse toward basic mathematics knowledge has a great potential to having good motivation but poor knowledge about mathematics. We can learn learners' interest from the keywords which learners enter in search box.

\section{CONCLUSION}

We developed two functions. One is to display inbound links on top of the webpages based on network data. This function enables learners to make their own learning paths not only toward basic mathematical knowledge but also toward advanced mathematical knowledge simply by clicking hyperlinks. The other is the button on top of webpage to access the graph drawing of a knowledge structure of mathematics. By utilizing these two functions learners are able to learn mathematics efficiently and effectively according their ability and curiosity.

\section{ACKNOWLEDGMENT}

We appreciate the student staffs who have contributed to the development of our e-learning website.

\section{REFERENCES}

[1] T. M. Duffy and D. H. Jonassen, "Constructivism: new implications for educational technology," Educational Technology, vol. 31, no. 5, pp. 7-12, May 1991.

[2] Y. Atif, R. Benlamri, and J. Berri. "Learning objects based framework for self-adaptive learning," Education and Information Technologies, vol. 8, no. 4, pp. 345-368, 2003.

[3] Y. Guo, X. B. Chen, and P. F. Li, "The effect of massive open online course on the universities in the western region," Advanced Materials Research, vol. 926, pp. 4657-4660, 2014.

[4] D. T. Seaton, Y. Bergner, I. Chuan, P. Mitros, and D. E. Pritchard, "Who does what in a massive open online course?" Communications of the ACM, vol.57, pp. 58-65, 2014.

[5] P. Brusilovsky, "Knowledge Tree: A distributed architecture for adaptive e-learning," in Proc. the 13th International World Wide Web Conference on Alternate Track Papers \& Posters, 2004, pp. 104-113.

[6] D. Shen, X. Wang, and H.-L. Chen, "Managing web-based learning resources for K-12 education: lessons learned from web analytics," in Proc. World Conference on e-Learning in Corporate, Government, Healthcare, and Higher Education, Las Vegas, Nevada, USA, 2008, pp. $470-475$.

[7] D. Ellis, "Google analytics as a tool in the development of e-learning artefacts: A case study," in Proc. Australian Society for Computers in Learning in Tertiary Education Annual Conference, Wellington, New Zealand, 2012, pp. 299-303.

[8] Yin, Chengiiu et al., "Learning by searching: A learning environment that provides searching and analysis facilities for supporting trend analysis activities," Journal of Educational Technology \& Society, vol. 16, no. 3, pp. 286-300, 2013

[9] A. Nakamura. KIT mathematics navigation. [Online]. Available http://w3e.kanazawa-it.ac.jp/math/

[10] A. Nakamura. KIT mathematics navigation. [Online]. Available $\mathrm{http}: / /$ translate.google.com/translate?hl=ja\&sl=ja\&tl=en\&u=http\%3A $\% 2 \mathrm{~F} \% 2 \mathrm{Fw} 3$ e.kanazawa-it.ac.jp\%2Fmath\%2Ftrans\%2Fcategory\%2Fi ndex.html.

[11] A. Nakamura, "Log analysis of mobile user behavior for a public-facing math e-learning site," GSTF Journal on Education, vol. 2, no. 2, pp. 38-42, Nov. 2013. 
[12] A. Nakamura, "Hierarchy construction of mathematical knowledge," Lecture Notes on Information Theory, vol. 2, no. 2, pp. 203-207, Jun. 2014.

[13] A. Nakamura, "Graph drawing of knowledge structure of mathematics," The SIJ Transactions on Computer Science Engineering \& its Applications (CSEA), vol. 2, no. 4, pp. 161-165, Jun 2014.

[14] A. Nakamura, "Graph drawing of knowledge structure of mathematics combined with knowledge level," INTED2015 Proceedings, pp. 2576-2579, 2015.

[15] Gephi - The open graph viz platform. [Online]. Available: http://gephi.github.io/

[16] JavaScript GEXF viewer for Gephi. [Online]. Available: https://github.com/raphv/gexf-js

[17] A. Nakamura. Graph drawing of knowledge structure of mathematics (English version). [Online]. Available: http://w3e.kanazawa-it.ac.jp/math/gexf-js-en/index.cgi

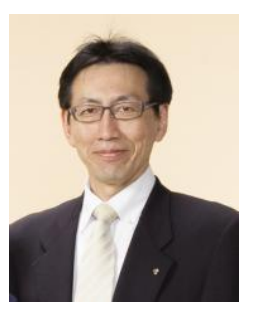

Akira Nakamura was born in Japan in 1961, and received his master of engineering degree from Osaka University in 1986 .

He joined Matsushita Electric Industrial Co., Ltd. (Panasonic) in 1986, and was engaged in the research and development of the lucid crystal display. $\mathrm{He}$ joined Kanazawa Institute of Technology in 2004, and presently holds the rank of professor. He has been engaged in teaching mathematics and physics, and in the research and development of e-learning.

Mr. Nakamura is a member of Japanese Society for Engineering Education, Japan Society for Educational Technology and Japanese Society for Information and Systems in Education. 